\title{
ESTUDIO HISTÓRICO SOBRE LA INTELIGENCIA; UN TÉRMINO INACABADO
}

\author{
A HISTORICAL STUDY ON INTELLIGENCE; AN UNFINISHED TERM
}

Griselda Jacqueline Morales Alarcón, Danú Alberto Fabré Platas, Sergio Vázquez Zavaleta

Universidad Veracruzana, México

Correspondencia: jaque10morales@gmail.com

\section{RESUMEN}

La inteligencia es un concepto primordial dentro de diferentes ámbitos, teniendo un impacto esencial dentro del educativo, en el cual se han elaborado distintos estudios desde el siglo pasado y el presente. Se ha plasmado conforme a las circunstancias y necesidades de la época, proporcionándole una esencia distinta al sujeto. Aunque en su mayoría es un estudio realizado por psicólogos, los postulados planteados son comprensibles dadas las exigencias que presentan en su realidad, la cual es tomada en cuenta para diseñar una definición característica de cada enfoque. De esta manera, las definiciones que se proporcionan comparten características, ya que una se retroalimenta y retoma de la otra, siendo así una construcción histórico-social en constante modificación. El presente trabajo se deriva de la tesis de licenciatura en pedagogía de la Universidad Veracruzana, denominado "Inteligencia emocional y las relaciones interpersonales de los estudiantes de pedagogía, generación 2016", de Morales Alarcón y Vázquez Zavaleta (2020).

Palabras clave: Inteligencia, educación, psicología. 


\section{ABSTRACT}

The term intelligence is a primary concept within different fields, having a significant impact within education, in which different studies have been carried out throughout the last century and the present. It has been defined according to the circumstances and necessities of its time, giving the subject a different essence. Although most studies are carried out by psychologist, the postulates are understandable given the demands they present in their reality, which is considered to design a definition unique to each point of view. This way, the definitions that are considered have a similar essence, because one retakes from the other, becoming in a way a social and historical construct. This work is derived from the undergraduate thesis in pedagogy, of the Universidad Veracruzana, titled "Emotional intelligence and interpersonal relationships of students of pedagogy, generation 2016" by Morales Alarcón and Vázquez Zavaleta (2020).

Key words: Intelligence, education, psychology.

\section{INTRODUCCIÓN}

A lo largo de la historia se han realizado estudios de diferente índole, siempre con el objetivo de encontrar respuestas a las interrogantes persistentes que se desarrollan en la cotidianidad de los sujetos. Para el caso de la inteligencia, no hay excepción, dado que se han abarcado un gama de posturas sobre el significado y definición, al igual que un indefinido número de características. En este sentido, algunos autores plasman dicho concepto como un suceso hereditario, otros más como punto medible, a su vez, el postulado de que el hombre posee diferentes inteligencias y por lo tanto todos los seres humanos son inteligentes en distintos ámbitos, continuando con aquella que considera la inteligencia emocional, y en los últimos años la denominada inteligencia artificial, por mencionar algunas. 
Para el caso de los espacios educativos suele denominarse inteligente a las personas que logran responder todos los ítems desarrollados por el docente, quien abarcó estos de acuerdo con el contenido visto en un trimestre o parcial de algunas asignaturas. Cuando el alumno no es capaz de responder exitosamente las preguntas relacionadas al grado escolar al que pertenece, es cuando se le denomina menos inteligente o incapaz que sus pares.

Sin embargo, existen limitaciones que le impiden lograr obtener todos los rubros, por ejemplo, cuando no tuvo las mismas oportunidades a alguien que ha venido estudiando y preparándose constantemente. Otra característica que se puede señalar es el hablar un segundo idioma como el inglés, pareciera ser que todo universitario debe de poseer a la perfección ya que lo ha practicado en los niveles previos que ha cursado. Sí bien el término obedece en la mayoría de las ocasiones al sector educativo, también se encuentra inmerso dentro de la cotidianidad.

Todo lo anteriormente mencionado, abre pauta para empezar a hablar sobre los diferentes autores que han plasmado su ideales a lo largo de la historia sobre la inteligencia, y como está comenzó a tener importancia desde el siglo pasado. No obstante, durante este lapso se han derivado varias posturas ocasionando la evolución del término y concibiéndolo de distinta manera. Por otra parte, es relevante comprender el significado de la inteligencia y sus distintas teorías para llevarlas a cabo en el proceso de enseñanza-aprendizaje, aulas y en la vida cotidiana.

La presente información, en un primer momento se deriva de la tesis de licenciatura "Inteligencia emocional y las relaciones interpersonales de los estudiantes de pedagogía, generación 2016", no obstante se recaban demás teorías y perspectivas sobre inteligencia, con el objetivo de analizar 
el cambio histórico y práctico que ha tenido el termino de inteligencia en la sociedad. Esto mediante una metodología de revisión sistemática, que de acuerdo con Beltrán (2005), "exige un método riguroso y explícito para la identificación, evaluación crítica y síntesis de la evidencia obtenida" (p. 61), esto con el propósito de revisar detenidamente la información que se encuentra dentro de la tesis, además de agregar otros artículos con la información necesaria y pertinente.

Con ello, se enfoca una técnica comparativa, que "tiene como objetivo la búsqueda de similitudes y disimilitudes (...) siendo la identidad de clase el elemento que legitima la comparación se compara entonces lo que pertenece al mismo género o especie" (Tonon, 2011, p. 2). De esta manera, se realiza una comparación exhaustiva de toda la información, permitiendo extraer los puntos esenciales de cada definición e identificar aquello que sea útil y benéfico para el progreso de este término.

\section{Perspectivas sobre la inteligencia}

En las siguientes líneas se analizarán las principales teorías que han dado pauta para el estudio de la inteligencia, mismas que se han concebido de diferente manera conforme pasan los años, posturas que han sido acuñadas por varios autores desde diferentes profesiones. Una vez descrito lo anterior, se abre pauta a lo que nos compete en el presente artículo. A lo largo de la historia universal el ser humano ha logrado responder a cuestiones complejas que previamente consideraba al observar un cambio en su alrededor. 


\section{La inteligencia como aspecto hereditario y medible en los siglos XIX y XX}

Los estudios y conocimientos han logrado puntos significativos por medio de los principales intelectuales en las diferentes ciencias. Un caso particular es lo sucedido con la publicación del "Origen de las Especies" de Darwin, con ello "se empezó a considerar al ser humano un poco más inteligente que los demás, se centró la atención en la herencia, en las diferencias individuales y en las emociones primarias" (Manrique, 2011, p. 92).

Siendo primo del autor anterior, Sir Francis Galton, citado por Sternberg (1987), propone la inteligencia del siguiente modo, "este concepto se hallaba íntimamente relacionado con la noción de que la inteligencia era, en gran medida una característica hereditaria" (p. 162). Como es el caso de los rasgos fenotípicos que dependen de los antecesores, y estos a la vez vienen de sus anteriores generaciones, por ejemplo, si ninguno de los padres tiene los ojos de color azul, pero sí el abuelo del padre, entonces tal rasgo fue heredado a través de él.

Continuando con lo anterior, en ciertas ocasiones la inteligencia se tiende asociar con el término Cociente Intelectual (C.I), emprendido por varios psicólogos entre los que cabe destacar a Alfred Binet, con los test de inteligencia, mismos que se desarrollaron para la mejora en la educación de los niños que se consideraban lentos en el aprendizaje, perspectiva considerada a finales del siglo XIX. Uno de los eventos a los que alude tal punto es el ingreso a una institución educativa, por ejemplo, la universidad al seleccionar la posible matricula de alumnos se aplica un examen que consiste en distintos puntos. Pero, el hecho de que algún estudiante por motivos desconocidos no logré acreditar dicho examen no define su nivel intelectual, sino se puede atribuir a distintos factores como la demanda que presenta dicha licenciatura. 
Regresando a las concepciones de los principales receptores en el campo de la psicometría, Binet determinó el test de inteligencia junto a Théodore Simon, autor perteneciente a la historia de la Psicometría, "cuando el niño tiene éxito en sus estudios, cuando obtiene buenas notas, no hay duda. El niño demuestra su inteligencia a través de sus acciones" (Binet, 1973, p. 115). Un estudiante que demuestra desempeño esmerándose en las actividades escolares, tratando de dar lo mejor de sí, ya está siendo inteligente porque conoce lo que debe realizar para obtener notas altas, mismas que lo llevarán a futuros estímulos por parte de la institución educativa.

Continuando con las palabras de Binet (1973), en referencia a la inteligencia denota lo siguiente:

La inteligencia, considerada independientemente de los fenómenos de sensibilidad, de emoción y de voluntad, es ante todo una facultad de conocimiento, dirigida hacia el mundo exterior y que trabaja para reconstruirlo en su totalidad a partir de los pequeños fragmentos de este que nos son dados (p. 116).

El propio autor hace diferencia entre aquella inteligencia derivada de los sentidos que posee el ser humano, mismos que son apropiados a lo largo de su vida. Ejemplificado en logros como diferenciar el frío de lo caliente por medio de los sentidos, asimismo la textura de todo lo papable y los diferentes sabores que conforman el contexto inmediato. En segunda instancia, deriva la emoción como parte fundamental del sujeto en su inteligencia. Por último, menciona la voluntad, aquella que viene del interior y va hacia el exterior tomando forma con lo que es proporcionando, siendo esta en la que mayor énfasis pone el autor. 
Cabe mencionar, que los autores del test psicométrico mencionan la Edad Mental (EM), que "partió de la hipótesis de que al menos durante la infancia y la adolescencia a medida que aumenta la edad cronológica, es decir, la edad real, aumenta también el nivel de inteligencia" (Cerdá, 1984, p. 125). Cuando los bebes están creciendo físicamente, experimentan cambios y necesidades que los orillan a una adaptación al ambiente en el que están interactuando. Por ello, en esta etapa se presencian avances significativos como: hablar y caminar, los cuales se van perfeccionando el resto de la vida.

Tal como lo menciona Cerdá (1984), la creación de escala estimativa sobre la inteligencia requiere un análisis preciso de la EM, siendo un rasgo difícil dado los tantos intentos por realizar hasta encontrar el idóneo. Aun así, con lo cientos de intentos previos probablemente se considere como inapropiado para una población mientras que para otros será ideal. Aspectos que demuestran un segundo inconveniente al tratarse de la inteligencia en la actualidad, donde persisten y estipulan teorías sobre las que se debe considerar como inteligencia y las que no.

Con referencia a lo anterior, se establece la siguiente cita:

Finalmente, una Edad Mental en sí misma no nos dice nada acerca del grado de capacidad de la persona. Una edad mental de 8 años significará que la inteligencia es normal de quien dio este resultado es un niño de 8 años, pero indicará una inteligencia muy superior a lo normal si el niño tiene 5, y una deficiencia mental si tiene 14 (Cerdá, 1984, p. 127). 
Destacando lo anterior, se hace énfasis en la importancia que juega la EM dentro de las capacidades de los sujetos, refiriéndose a esta como un punto indicativo entre la inteligencia y la edad cronológica del individuo. Estas aproximaciones hacen referencia si una persona es superdotada o si existe alguna deficiencia. Ante esto se encuentran casos de personas que ingresan con una edad inferior en Educación Superior, ya que los conocimientos que poseen son superiores a los sujetos de su misma edad. Por el contrario, existen casos sobre una deficiencia, al estar por debajo de los resultados esperados, pese a las diferencias que se realizan no excluyen las capacidades que tenga la persona.

Por otra parte, el análisis factorial es una técnica estadística utilizada para explicar la correspondencia que existe entre variables observadas y no observadas, en la última deben de ser con un rango menor. A pesar de su origen en la psicometría, también se utiliza para otras ramas del conocimiento como las ciencias del comportamiento y sociales.

A principios del siglo XX el psicólogo de origen inglés Charles Edward Spearman propone la teoría Bifactorial donde plasma dos puntos que constituyen la inteligencia humana. Parafraseando a Pérez y Medrano (2013), la primera consistía en un Factor General (G), que se deriva de un punto que posee toda persona y puede ser hereditario o no, haciendo presencia en la conducta del ser humano durante toda su vida. Por otra parte, se encuentra el Factor Especial, por su connotación en inglés (S), que establece aquellas actitudes o habilidades específicas que posee cada individuo ante una situación dada.

Continuando con lo planteado por el psicólogo e ingeniero mecánico estadunidense Louis León Thurstone, citado por Villamizar y Donoso 
(2013), quien con el análisis factorial descubrió siete puntos, los cuales son; Fluidez verbal, Compresión verbal, Actitud espacial, Rapidez receptiva, Razonamiento inductivo, Aptitud numérica y Memoria. Además, "propuso realizar a cada persona un 'perfil' aptitudinal, que expresara las diferencias entre dos individuos con igual coeficiente intelectual” (p. 411).

A su vez con los estudios de comprender, medir e interpretar la inteligencia, el psicólogo estadunidense Joy Paul Guilford, citado por Krumm, Filipetti y Bustos (2013), sobresale por los diferentes estudios implementados acerca de la inteligencia, en especial con el modelo "Estructura de la inteligencia", que sugiere la inteligencia para "comprenderse en términos de un cubo que representa la intersección de tres dimensiones (a) las operaciones mentales, (b) los contenidos mentales y (c) los productos mentales" (p. 1533).

Retomando los estudios realizado por Binet acerca de los tests psicométricos, William Lewis Stern un psicólogo, filósofo e intelectual alemán, concibe el término del Cociente intelectual (CI). Se caracteriza por la relación entre la edad mental y edad cronológica, como lo menciona Cerdá (1984), "el cociente intelectual es el resultado de dividir la Edad Mental por la Edad Cronológica y, para evitar decimales multiplicarlo por 100 " (p.127). Esta fórmula para obtener el CI de las personas es aplicado acorde a los parámetros y edad que posee el individuo. Sin embargo, no solo mide aspectos teóricos surgidos a lo largo de la historia o datos y fechas importantes, sino la capacidad de resolver las problemáticas en el momento oportuno y adecuado.

Por otra parte, Raymond Bernard Cattell, psicólogo británico propone dos factores para la conceptualización de la inteligencia, a pesar de la infinidad 
de intentos diseñados previa y posteriormente sobre la inteligencia del ser humano. Los dos puntos son la Inteligencia Fluida (Gf), teniendo como característica aquella capacidad que posee el ser humano para enfrentar aquellas situaciones nuevas, sin que sus conocimientos antecesores intervengan en el proceso; mientras que el otro punto definido como Inteligencia Cristalizada (Gc) muestra lo contario.

A pesar de todos los avances conseguidos aún faltaba por indagar en otros horizontes, situación semejante que se percibe en la actualidad. Siendo un laberinto sin salida, donde se encuentra demasiada información en torno a un tema o problemática. Por ejemplo, al realizar un trabajo de investigación donde se requiere una amplia búsqueda de referentes teóricos que permitan aludir a la problemática tratada. Sin embargo, con el tiempo transcurrido se alienaron ciertos conceptos para cada aspecto, así como el de "idiota para aquellos que no sabían nada, imbécil para los que se encontraban en un nivel medio y estúpido para un nivel un poco debajo por lo que sería considerado un rango normal de inteligencia" (Cattell, 1987, p. 23, traducción propia).

Para el siglo XX surge una proliferación de teorías en torno a la inteligencia, siguiendo las categorías que, lejos de beneficiar al sujeto, lo catalogaban en niveles considerados inferiores a los demás por el hecho de salir con un porcentaje inesperado en las pruebas psicométricas que la media, ocasionando estandarización en la memoria. Explicado en palabras del autor la "inteligencia es la adaptabilidad a nuevas situaciones. Cualquier comparación de lo que comúnmente es considerado como un ser inteligente o no, apunta a que el primero es capaz de seguir sus instintos en circunstancias que han sido introducidas de manera inusual" (Cattell, 1987, p. 12, traducción propia). El sujeto merecedor de tal punto necesita 
poseer aquella habilidad que le permita indagar nuevos campos a pesar de ser desconocidos y poco a poco adaptarse a ellos.

Prosiguiendo con el análisis de las teorías, el psicólogo ingles afirma que "Thurstone no solo comprobó la existencia de distintas habilidades primarias, pero también demostró que una sola habilidad general podría ser considerada retrasada, en un sentido de no tener una especialización" (Cattell, 1987, p. 30, traducción propia). Por otra parte, el psicólogo estadunidense Robert Jeffrey Sternberg, conocido por la Teoría Triárquica de la Inteligencia que consiste en una inteligencia analítica, practica y creativa establece los siguiente:

La inteligencia nos proporciona medios para gobernarnos a nosotros mismos, de manera que nuestros pensamientos $\mathrm{y}$ acciones se organicen de manera coherente y responsable con respecto tanto a nuestras necesidades internas como con respecto a las necesidades del medio ambiente (Sternberg y Detterman, 1992, p. 33).

Lo vincula entre la exigencia extrínseca e intrínseca, a manera que lo planteado en la mente sea congruente con el exterior en el que se convive. Para realizar algo, primero pasa a ser idealizada en los pensamientos para posteriormente trasladarlo a la realidad. Inconscientemente se planean las labores que regirán el día señalando tiempos y formas en que serán ocupados, pero no siempre sucede de esa manera dado que surgen imprevistos que alteran lo diseñado previamente. Ante esto se buscan alternativas posibles, pensando y actuando a la par para que no exista una incoherencia entre ambos puntos. 
Continuando con las ideas de los autores, desglosan que "la inteligencia no reside de modo exclusivo dentro del individuo, sino que más bien se halla en las respuestas que el individuo da a las exigencias que le plantea su contexto social, cultural o de otro tipo" (Sternberg, 1987, p. 37). Aspecto por el cual la educación debe ser contextualizada dependiendo de las circunstancias que enfrente cada territorio. Dejando la idea sobre el funcionamiento de un modelo en concreto en toda una región, para afirmar ello, se tendrán que tratar primeramente puntos económicos, culturales, políticos y educativos que rigen tal contexto para posteriormente aplicar o adaptar las propuestas que ya han encontrado resultados positivos en otros lugares.

Pasando a otra concepción sobre la inteligencia, el psicólogo estadunidense Douglas K. Detterman investigador de Inteligencia y Discapacidad Intelectual menciona lo siguiente "la inteligencia como un sistema complejo integrado por numerosos procesos cognitivos independientes. Estos procesos contribuyen a la aparición de un factor general" (Sternberg y Detterman, 1992, p. 28). En ese sentido, dicho termino no debe ser determinado por un rasgo, sino que intervienen varios. Tampoco es proporcionaruna lista de requisitos que establezcan a una persona inteligente o no. Ante la conceptualización del autor, encara que la inteligencia no es sinónimo de obtener altas notas en las pruebas objetivas, sino de múltiples factores que intervienen en el proceso.

A su vez el mismo autor se refiere a que "la inteligencia puede definirse mejor como un conjunto limitado de aptitudes independientes que actúan como un sistema complejo" (Sternberg y Detterman, 1992, p. 76). Los sujetos son poseedores de diferentes habilidades y capacidades, ante tal aspecto, poseedores de diferentes aptitudes que proporcionan una ayuda 
a desempeñarse en el contexto que los rodea. Al referirse a limitado, es porque los seres humanos a pesar de toda la capacidad que los respalda no pueden abstraer todas las habilidades encontradas. Quizás podrán tener un gran número que facilite sus actividades cotidianas, sin embargo, las que obtengan y vaya perfeccionan a lo largo de su estadía vivencial serán suficientes para lograr la denominada "Inteligencia".

\section{La inteligencia desde diferentes teorías: Motivación, Desarrollo cognitivo y Aprendizaje sociocultural}

Llegados a este punto, se retoma la interpretación de inteligencia del psicólogo, Jean Piaget, quien deriva las siguientes líneas sobre la inteligencia, concibiéndola como una adaptación sobre su contexto ya sea físico o de pensar, siendo a la vez un vínculo entre la persona y su contexto. En este sentido "la vida en efecto es una creación continua de formas cada vez más complejas y un establecimiento en equilibrio progresivo entre estas formas y el medio" (Piaget, 1994, p. 14).

Por consiguiente, parafraseando a Piaget (1994), la inteligencia es dependiente de ciertos factores como la racionalización, considerada como organización y la experiencia que se compone de la adaptación no solo del espacio, también retoma las costumbres, actuar y concebir un punto en concreto. En este sentido, ser inteligente no implica concederle todo el crédito a la razón o a la experiencia, puesto que son dos factores que trabajan colaborativamente para constituir un hombre completo en su intelecto. Lejos de ser dos puntos opuestos, la inteligencia y los sentimientos son pautas que tiene el ser humano "y las que se refieren a las ideas o a las cosas: pero cada una de esas conductas intervienen los mismos aspectos afectivos y cognoscitivos de la acción, aspectos siempre unidos y que en ninguna forma caracterizan facultades independientes" (Piaget, 1983, p. 16). 
Finalizando las concepciones de Piaget (1983), "la inteligencia aparece, en suma, como una estructuración que imprime ciertas formas a los intercambios entre el o los sujetos y los objetos que lo rodean tanto si están cerca como si están lejos" (p. 183). Tomando en cuenta que la inteligencia tiene una organización diferente en cada ser, cada persona tiene una historia diferente que le permite desarrollar ciertas habilidades cognitivas, como lo mencionan Ausubel, Hanesian y Novak (2005).

Un factor que mencionan los autores acerca de la inteligencia es que "a medida que aumenta la edad la inteligencia manifiesta un grado considerable y creciente de estabilidad a través del tiempo (especialmente durante los años escolares) no significa que sea completamente estable, inmutable o insensible a los factores del ambiente" (Ausubel, et al, 2005, p. 229). Tomando en cuenta estos tres aspectos, se ve que la composición de una inteligencia es variada y nunca va a ser igual, es prácticamente imposible que dos personas tengan un mismo desarrollo. En primera instancia se tiene el aspecto genético, cada ser humano tiene una composición biológica en la cual se encuentra toda la información personal, en este caso la composición genética tiene dos vertientes, una universal y otra personal.

Bajo otro punto de vista, el psicólogo estadunidense, impulsor de la Psicología Humanista, Abraham Maslow (1991), menciona la definición del coeficiente intelectual y sobre las personas que podrían ser catalogadas con dicho parámetro. Además, puntualiza que este punto no se dirige a otros factores, más que tecnológicos. "Sin embargo, una psicología que se limita a si misma de tal manera pierde los aspectos más importantes del hombre (sabiduría, conocimiento, insight, compresión, sentido común, buen juicio) en favor del C. I. sólo porque tecnológicamente es más satisfactorio" (p. 267). 
Por otra parte, el ser inteligente según los rubros establecidos, no es indicio de poseer la capacidad para manejar a la perfección aspectos de moralidad, conciencia, sabiduría, sentido común. Por ello Maslow (2008), no solo proporciona el conocimiento a las personas autorrealizadas, de acuerdo con la pirámide que propone en la teoría del humanismo, o a las personas vastas en inteligencia, sino las que a continuación menciona:

Mi impresión experimental es que tengo más probabilidades de descubrir un conocimiento de la trascendencia no solo en personas autorrealizadoras, sino también en gente altamente creativa, inteligente, de carácter fuerte, en líderes y directores poderosos y responsables, en personas excepcionalmente buenas (virtuosas) y en personas "Heróicas" que han superado la adversidad que se han fortalecido en vez de debilitarse (pp. 335-336).

Derivado de las perspectivas que brinda Maslow (2007) sobre la inteligencia indica que, si se es poseedor de varios puntos, incluyendo ser inteligentes, se deben explotar para cubrir las necesidades propias y las que impone la sociedad. Entre los que cabe mencionar; concluir los niveles educativos obligatorios; educación básica, media superior y superior.

Estas se denominan capacidades que los individuos tienen, si bien algunos demuestran ser más aptos para otras, esto no indica que los demás no logren llevarlas a cabo, o que sean aptos para todas. Según Maslow (2007) se ofrece lo que se tiene, no se promete algo indisponible en la persona, a la vez que se realizan tareas con las herramientas que se tienen al alcance. Las personas con ciertas habilidades las explotan, aquellas que siente aprecio por alguien más lo difunden, las diferentes capacidades obligan a ser utilizadas y "sólo cesan en su exigencia cuando son realmente 
utilizadas de manera suficiente. Es decir, las capacidades son necesidades $\mathrm{y}$, por tanto, son también valores intrínsecos. En la misma medida en que difieran dichas necesidades, diferirán también los valores” (p. 108).

Por otra parte, Maslow (2007), sigue señalado la importancia de utilizar la inteligencia, ya sea en el trabajo, escuela o en casa, mientras sea de la manera correcta, dado que un estudiante puede ser catalogado inteligente con todas las capacidades por nómbralo de tal manera. Asimismo, comprende los conocimientos rudimentarios establecidos en la sociedad sobre un determinado tema, "lo mismo cabe decir de la inteligencia, del útero, de los ojos, de la capacidad amorosa. Las capacidades exigen ser usadas y tan sólo cesan en su exigencia cuando son usadas adecuadamente. Es decir, las capacidades son también necesidades" (p. 139).

Los aspectos que rigen cuando una persona merece ser nombrada como inteligente, no son premiados por lo establecido de una sola persona o dicho de un ser que se le ocurrió tal diferencia. Estas oposiciones son vinculadas por lo establecido en la mayoría de la población de un contexto determinado, aquel ser humano que merezca estar en un grupo o en otro. En dado caso lo que es argumentado en su minoría no tiene valor a diferencia de aquello que es promulgado por unanimidad. En este sentido Vygotsky, “estableció que el estudio de la inteligencia se ve premiado por las diferencias entre las culturas y las prácticas sociales, más que por las diferencias entre los individuos" (Trujillo y Rivas, 2005, p.11). Cabe mencionar que en el rango de inteligencia varía de acuerdo con la cultura. Es decir, un individuo puede ser nombrado como inteligente en la cultura mexicana mientras que la japonesa no y viceversa. 


\section{La inteligencia y sus diferentes interpretaciones durante las últimas dos décadas}

Por consiguiente, la “inteligencia humana más allá de los aspectos cognitivos e intelectuales que resalte la importancia. Existen habilidades más importantes que la inteligencia académica a la hora de alcanzar un mayor bienestar laboral, personal, académico y social" (Pacheco y Fernández-Berrocal, 2004, p.2). Como se abordó en apartados previos, la obtención de resultados favorables en una prueba de conocimientos no es garantía de una estabilidad económica o social para las personas. Dentro de un grupo de varios puntos algunos de ellos son la empatía, convivencia con los demás, manejo de conocimiento en espacios públicos, responsabilidad, puntualidad, interés por aprender, entre otros más. Los cuales llevaran a crear las posibilidades académicas, económicas y sociales de la persona.

En contraste con lo anterior Valdés, Vera y Carlos (2013) precisan que "los modelos orientados a las capacidades sostienen que las aptitudes intelectuales se explican por la presencia de una inteligencia superior originada fundamentalmente por factores genéticos, por lo que se evidencian desde la infancia y se mantienen estables en el tiempo" (p.87). En este sentido cuando un estudiante o persona desde una temprana edad muestra indicios intelectuales necesarios o en su caso sobresalientes los mantiene conforme pasa el tiempo. A su vez si el niño tiene padres inteligentes podrá hacer uso de esta genética obteniendo los mismos resultados que sus progenitores. Sin embargo, se resaltaría en buena medida mejor, si tal privilegio se concibiera a la manera en cómo los padres enseñan a los niños, tiempo y dedicación que emplean para que sus hijos adquieran las bases rudimentarias en su formación escolar. 
Haciendo alusión a los puntos que se enfocan en el estudio de la inteligencia existen aquellos caracterizados "por su énfasis en cuantificar y ordenar las habilidades intelectuales de las personas, en la confianza en los test generales de inteligencia como base para sus datos y en el uso del análisis factorial para analizarlos" (Molero, Saiz y Martínez, 1998, p. 13). Mismos que pretender brindar un mejor resultado de los estudios psicométricos, ya que a pesar del acuerdo o desacuerdo por establecer este tipo de pruebas se tiene que partir de un punto donde se conozcan los aspectos que poseen los estudiantes. De esta manera se tiene la noción desde que ángulo se debe de enseñar y desde que peldaño se debe continuar según los estándares de conocimientos que tengan de niveles educativos previos.

Por lo que refiere al estudio de la Inteligencia, en la década de los 20 del siglo pasado Thorndike presenta un artículo denominado: "La inteligencia y sus usos" abarcando "qué significa inteligencia e introduce el componente social en su definición. En dicho artículo señala la existencia de tres tipos de inteligencia: la inteligencia abstracta, la inteligencia mecánica y la inteligencia social" (Molero, et al., 1998, p. 15). Misma que abre pauta para posteriores estudios entre los que cabe destacar, la teoría de inteligencias múltiples de Gardner y la inteligencia emocional de Goleman, ya que establece la inteligencia social, hecho que instituye la relación con los demás. De esta manera queda concretada como la eficiente de pensar, hacer y relacionarse, mismas que son parte del desarrollo y de la cotidianidad de las personas.

Molero, et al. (1998), realizaron un bosquejo sobre la conceptualización de la inteligencia, mismas que presentan rasgos significativos y diferentes, en donde todas son rescatables para establecer el propio concepto, ya que ninguno está en lo incorrecto, simplemente concibió las características 
de acuerdo con su formación y el contexto en donde se encontraba, sugiriendo las mejores opciones adaptadas a su realidad. A continuación, se presentan las cuatro posturas, "el poder de dar buenas respuestas desde el punto de vista de la verdad o el hecho" (Thorndike);"la capacidad de pasar a un pensamiento abstracto" (Terman); "la capacidad de adaptarse adecuadamente a la vida en situaciones relativamente nuevas" (Pintner); "la capacidad para adquirir capacidad" (Woodrow). Ninguna de las definiciones que se presentaron, agrado en sus totalidad a los investigadores.

Pasando a otro aspecto, Scarr y Carter-Saltzman, en Molero, et al, (1998), afirman al momento de hablar sobre inteligencia a través de "diferentes niveles que no están necesariamente relacionados entre sí. El término puede aplicarse o aplicarse mal a cuatro niveles: a un nivel cultural, a un nivel de organización social, en pequeños grupos y finalmente a un nivel individual" (p. 22). Los cuatros niveles plasman los tipos de interacción que tienen los sujetos en su entorno inmediato. De tal modo, abarcar los cuatro niveles es una cuestión complicada, ya que cumplir los estándares de inteligencia en tantos rubros, a partir de lo general a lo particular para al final complementar la definición. Es una tarea que requiere verificar la viabilidad en todos los puntos, ya que puede estar siendo eficaz en algunos mientras que los demás no.

Continuando con la conceptualización de la inteligencia para Marina (1993) significa aquella que es "transfigurada por la libertad. La inteligencia creadora obra haciendo proyectos. El más arriesgado proyecto de la inteligencia es crear un modelo de inteligencia, es decir, de sujeto humano, es decir de humanidad" (p.209). El mismo autor proporciona que la inteligencia, rasgo encontrado en los seres humanos "no es el eficaz dinamismo de una computadora, sino la costosa búsqueda de la libertad por 
parte de un sujeto que sabe manejar información, pero que ha de hacerlo bregando contra la dificultad, el cansancio y las distracciones (Marina, 1993, p.304). Las personas desarrollan el intelecto conforme eventos presentados que los forman, moldeándolos conforme interactúa con sus semejantes y objetos.

Prosiguiendo con lo que compete, Ardilla (2011), propone que "las pautas que dirigen al concepto de inteligencia, como capacidad de solucionar problemas, de razonar, de adaptarse al ambiente, han sido altamente valoradas lo largo de la historia" (p. 98). además de que hoy día se despliega dicho termino como parte de las personas, ya que independiente de su grado académico, contexto, conocimientos en las disciplinas. Todos poseen inteligencia o en su caso inteligencias, mismas que les permiten trabajar en los sectores en donde estén inmersos. Sin embargo, Ardilla (2011) señala independiente del grado de mayor o menor inteligencia, hace presencia en los animales. Aspecto señalado en ciertas ocasiones al llamar a una mascota y este entiende lo dicho por su amo, proclamando la esencia del intelecto en él.

Con referencia a lo anterior Pacheco y Fernández-Berrocal (2004) plasman que, "las personas emocionalmente inteligentes no sólo serán más hábiles para percibir, comprender y manejar sus propias emociones, sino también serán más capaces de extrapolar sus habilidades de percepción, comprensión y manejo a las emociones de los demás" (p. 6). Desde esta postura dicha inteligencia no solo será benéfica en el sector académico sino también en la vida personal y social, rasgos que son parte de su vida cotidiana. Constado la conceptualización de Mayer y Salovey, expuesta por Salazar-Ceballos (2018), la inteligencia puede ser aquello que permite "percibir, valorar y expresar emociones con exactitud, la habilidad para 
acceder y/o generar sentimientos que faciliten el pensamiento; la habilidad para comprender emociones y el conocimiento emocional y la habilidad para regular las emociones promoviendo un crecimiento emocional e intelectual" (p.249).

\section{La inteligencia y las inteligencias de la actualidad}

Por otra parte, el psicólogo estadunidense Howard Gardner, pionero en las inteligencias múltiples, señala que la inteligencia no se deriva de obtener buenos resultados en aspectos de matemáticas, física o química, o del establecer una inteligencia universal, como se había manejado antes de la aparición de dicha teoría, de las cuales se derivan las siguientes: inteligencia lingüística (o verbal-lingüística), lógico matemática, visual, corporal (o quinestésica), musical, naturista, intrapersonal e interpersonal.

De esta manera se encuentra la teoría de las inteligencias múltiples como; "inteligencia como la capacidad de resolver problemas o de crear productos que son valorados en uno o más contextos culturales" (Gardner, 2001, pp. 44-45). La inteligencia es una capacidad que posee el ser humano y es una herramienta para resolver los problemas que perjudican a su persona y a la sociedad. Como seres evolucionados, se cuenta con el aspecto cognitivo avanzado que permite el poder crear y mejorar la sociedad en la que se vive, como menciona Gardner, la inteligencia no es una sola universal, no se debe clasificar a los seres en inteligentes o no con un solo criterio ya que el aspecto cognitivo abarca muchas áreas de la personalidad.

Para finalizar el aspecto de la inteligencia, solo hasta este momento, dado que, en un porvenir es probable el surgimiento de nuevas maneras de concebir el término. De acuerdo con los cambios presentados en la sociedad a lo largo de la historia se retoma a la inteligencia artificial 
como parte de este bosquejo, misma que es definida de la siguiente manera; "inteligencia artificial es tomar decisiones lógicas, algo que los seres humanos han olvidado, específicamente quienes tienen el poder de decisión sobre la mayoría, poder de decisión no solo política sino también en todos los niveles de la educación" (Salazar-Ceballos, 2018, p. 249). En donde la creación de la mencionada inteligencia es con el objetivo de lograr que "solucione los problemas de "muchos seres humanos" o creado por otros seres humanos, muchos creen ser inteligentes y actúan sin prever los efectos de sus acciones, solamente por un fin inmediato, una especie muy ansiosa. educación (Salazar-Ceballos, 2018, p. 250).

\section{CONCLUSIÓN}

A manera de conclusión, una vez indagadas algunas conceptualizaciones se postula que ninguna es errónea, ya que cada uno lo define de acuerdo con las circunstancias de formación, vida y contexto. Partiendo desde este punto y retomando todos los aspectos se integra a la inteligencia como aquella gama de habilidades que permite a la persona resolver las problemáticas presentadas de manera apropiada y coherente, haciendo uso del razonamiento y de la idealización sobre las posibilidades que puede tratar.

De acuerdo con el referente empírico, la palabra inteligencia se escucha principalmente en espacios educativos. Lo anterior se ve ejemplificado al proporcionar un valor a cada actividad o acción que el individuo demuestre al término del ciclo escolar, sí logran cumplir con todos los rubros establecidos en el encuadre obtiene una nota de excelencia, que en ciertas ocasiones se denota con una calificación de diez. Este número indica el cumplimiento de lo establecido en las primeras clases del curso o en su caso que proporciono más de lo establecido. 
Pese a que se utiliza en instituciones educativas, no es el único lugar donde hace presencia dicha palabra, dado que se puede manejar en diferentes espacios, en lugares públicos, hogar o en instituciones de diferente índole. En la mayoría de las ocasiones, se asocia con obtener buenas calificaciones o saber en demasía alguna temática abordada en un periodo determinado. Se habla tanto de inteligencia como un valor cuantificable que se ha llegado a creer que solo se asocia con aspectos de calificaciones. Asimismo, con un valor de excelencia o saber más que el otro en ciertos puntos.

Por consiguiente, la inteligencia es un término importante en el sector educativo, al final de un periodo se tendrá una calificación en un portal o documento que avale el tiempo y acreditación de una materia. Proceso en Educación Básica, Media Superior y Superior en todas las modalidades, llevado a cabo por el personal administrativo o en su caso por el mismo docente.

Como se ha mencionado previamente, la inteligencia es un término que se ha manejado a lo largo del tiempo por diferentes personajes que han incursionado en dicho ámbito. Mismos, que han estudiado en este campo, formulando teorías que competían a las circunstancias con las que interactuaban y recabando información con investigaciones previas. Cabe mencionar que en ciertas ocasiones se oponían a lo que formulaban las anteriores, proporcionando una antítesis, tratando de crear nuevas concepciones e ideales de acuerdo con las realidades a las que se enfrentaba la sociedad.

Al realizar un análisis que permitiera sustentar la presente investigación se encontró un sinfín de teorías que permean sobre la conceptualización y origen de inteligencia desde diferentes enfoques, donde cada autor propone 
el significado personal según las circunstancias y referentes donde se esté sustentando o, por el contrario, para contraponer lo que su antecesor argumentó por medio de una serie de ideales que haya seguido. En este sentido, a continuación, se abordan las posturas en un orden de antigüedad.

Primero los estudios de Darwin sobre el origen de las especies, de manera explícita menciona la inteligencia. Seguido de los puntos de Galton, Binet, Stern, Spearman, Thurstone, Guilford, Cattell, Sternberg y Detterman. Siendo quienes se dedicaron al estudio de la inteligencia a excepción de los primeros dos. Por otra parte, por medio de las diferentes teorías del aprendizaje, con referencia a los autores Piaget y Ausubel. También se retomaron los postulados de la corriente del humanismo con Maslow y finalmente la teoría de las inteligencias múltiples de Gardner.

\section{REFERENCIAS}

I Ardila, Rubén. (2011). Inteligencia ¿Qué sabemos y qué nos falta por investigar? Revista de la Academia Colombiana de Ciencias Exactas, Físicas y Naturales, 35(134), 97-103. http:/www.scielo.org.co/scielo. php?script=sci_arttext\&pid=S0370-39082011000100009

I Ausubel, D. P., Novak, J, D. \& Hanesian, H. (2005). Psicología Educativa. Un punto de vista Cognoscitivo (segunda ed.). México: Trillas.

\Beltrán G., O. A. (2005) Revisiones sistemáticas de la literatura. Revista Colombiana de Gastroenterología, $20(1)$, pp. $60-69$.

Binet, A. (1973). Ideas modernas sobre los niños. Paris: Flammarion.

I Cattell, R. B. (1987). Intelligence: its structure, growth and action. Nueva York; Elsevier Science Publisher. https://www.elsevier.com/books/intelligence-its-structure-growth-and-action/cattell/978-0-444-87922-6

I Cerdá, E. (1984). Psicometría general (Tercera Edición). Barcelona: Herder.

I Extremera Pacheco, Natalio, \& Fernández-Berrocal, Pablo. (2004). El papel de la inteligencia emocional en el alumnado: evidencias empíricas. Revista electrónica de investigación educativa, 6(2), 117.

1 http://www.scielo.org.mx/scielo.php?script=sci_arttext\&pid=S1607-40412004000200005

I Extremera Pacheco, Natalio, \& Fernández-Berrocal, Pablo. (2004). El papel de la inteligencia emocional en el alumnado: evidencias empíricas. Revista electrónica de investigación educativa, 6(2), 117.

1 http://www.scielo.org.mx/scielo.php?script=sci_arttext\&pid=S1607-40412004000200005

\ Gardner, H. (2001). La inteligencia reformulada. Las inteligencias múltiples en el siglo XXI. Barcelona, España: Paidós Ibérica. 
I Krumm, G., Filippetti, V. A. \& Bustos, D. (2013). Inteligencia y creatividad: correlatos entre los constructos a través de dos estudios empíricos. Universitas Psychologica, 13 (4), pp. 1531-1543. https://www.redalyc. org/pdf/647/64735353024.pdf

I Manrique Tisnés, H. (2011). La contribución de Darwin al surgimiento de la psicología evolutiva. Revista de psicología Universidad de Antioquia, 3(2), pp. 83-98. http://pepsic.bvsalud.org/scielo.php?script=sci_ abstract\&pid=S2145-48922011000200007\&lng=pt\&nrm=iso

I Marina, J. A. (1993). Teoría de la Inteligencia Creadora. Barcelona, España: Anagrama.

I Maslow, A. (1991). Motivación y Personalidad. Madrid, España: Editorial Díaz de Santos.

I Maslow, A. (2007). El hombre Autorrealizado. Hacia una psicología del ser. Barcelona, España: Kairós.

I Maslow, A. (2008). La personalidad creadora. Barcelona, España: Kairós.

$\checkmark$ Molero Moreno, C., Saiz Vicente, E. \& Martínez, C. E. (1998). Revisión histórica del concepto de inteligencia: una aproximación a la inteligencia emocional. Revista Latinoamericana de Psicología, 30(1), pp. 11- 30. https://www.redalyc.org/pdf/805/80530101.pdf

I Morales Alarcón, G. J. \& Vázquez Zavaleta, S. (2020). Inteligencia emocional y las relaciones interpersonales de los estudiantes de pedagogía, generación 2016. Tesis de licenciatura, Universidad Veracruzana, Xalapa, Veracruz.

I Pérez, E. \& Medrano, L. A. (2013). Teorías contemporáneas de la inteligencia. Una revisión crítica de la literatura. Revista Latinoamericana de Ciencia Psicológica, 5(2), pp. 105 - 118. https://www.redalyc.org/ pdf/3331/333129928007.pdf

I Piaget, J. (1994). El nacimiento de la inteligencia en el niño. México: Grijalbo.

I Salazar-Ceballos, A. (2018). La inteligencia vs la inteligencia humana. Duazary: Revista Internacional de Ciencias de la Salud, 15(3), pp. 249-250. https://revistas.unimagdalena.edu.co/index.php/duazary/article/ view/2412

I Sternberg, R. J. (1987). La naturaleza de la Inteligencia humana y su medición (Primera ed.). Barcelona, España: Paidós Ibérica

I Sternberg, R. J. \& Detterman, D. K. (1992). ¿Qué es la inteligencia? Enfoque actual de su naturaleza y definición. Madrid: Pirámide.

1 Thorndike, L.L. (1920). Intelligence and its uses. Harper's Magazine, 140 (1), pp. 227 - 235.

I Tonon, G. (2011). La utilización del método comparativo en estudios cualitativos en ciencias políticas y ciencias sociales. KAIROS, 15 (27), pp. 1 - 12.

1 Trujillo Flores, M. M. \& Rivas Tovar, L. A. (2005). Orígenes, evolución y modelos de inteligencia emocional. INNOVAR. Revista de Ciencias Administrativas y Sociales, 15 (25), pp. 9 - 24. http://www. scielo.org.co/pdf/inno/v15n25/v15n25a01.pdf

I Valdés Cuervo, Ángel Alberto, Vera Noriega, José Angel, \& Martínez, Ernesto Alonso Carlos. (2013). Variables que diferencian a estudiantes de bachillerato con y sin aptitudes intelectuales sobresalientes. Revista electrónica de investigación educativa, 15(3), 86 - 97. http://www.scielo.org.mx/scielo. php?script $=$ sci_abstract\&pid=S1607-40412013000300006\&lng=es\&nrm=iso

I Villamizar, G., \& Donoso, R. (2013). Definiciones y teorías sobre inteligencia. Revisión histórica. Psicogente, 16 (30), pp.407 - 423. http://revistas.unisimon.edu.co/index.php/psicogente/article/view/1927 
Envío a dictamen: 2 de noviembre de 2020

Reenvío: 29 de enero de 2021 Aprobación: 15 de febrero de 2021

Griselda Jacqueline Morales Alarcón. Licenciada en pedagogía por la Universidad Veracruzana . Asistente de investigación en el Instituto de Investigaciones y Estudios Superiores Económicos y Sociales (IIESES), Universidad Veracruzana. Correo electrónico: jaque10morales@gmail.com

Danú Alberto Fabré Platas. Doctor en sociología por la Benemérita Universidad Autónoma de Puebla. Investigador de tiempo completo en el Instituto de investigaciones y Estudios Superiores Económicos y Sociales (IIESES), Universidad Veracruzana. Líneas de investigación: Dinámicas culturales y reconfiguraciones socioterritoriales, Sociedad, cultura y educación, Medio ambiente y desarrollo regional-territorial, Gestión del conocimiento: fundamentos epistemológicos, construcción y distribución social. Miembro del Sistema Nacional de Investigadores. Nivel 1. Correo electrónico: danufabre@gmail.com

Sergio Vázquez Zavaleta. Estudiante de la Facultad de pedagogía de la Universidad Veracruzana, campus Xalapa. Correo electrónico: sergvazzav@gmail.com 